\title{
Potential clinically significant life-threatening drug-drug interactions of lopinavir and ritonavir used in the treatment of COVID-19
}

\author{
Mohitosh Biswas ${ }^{1}$ \\ ${ }^{1}$ University of Rajshahi
}

July 2, 2020

\begin{abstract}
Aims: Lopinavir (LPV) and ritonavir (RTV) is using as combination therapy for combating COVID-19 in considerable proportion of patients. However, the potential clinically significant life-threatening drug-drug interactions (DDIs) of this combination antiviral therapy used in the treatment of COVID-19 patients is not systematically reviewed. It was aimed to identify severe DDI pairs of LPV/RTV from international resources predicted to cause life-threatening adverse drug reactions (ADRs) and cross-compared between the resources. Methods: Severe DDI pairs predicted to cause life-threatening ADRs were identified from the FDA and Liverpool COVID-19 prescribing information of LPV/RTV. Results: In total, 62 severe DDI pairs were identified from the FDA and Liverpool COVID-19 drug interactions resources predicted to cause life-threatening ADRs in patients with COVID-19. Of these, seven unique DDI pairs were identified from the FDA only whereas 45 unique DDI pairs were identified from the Liverpool COVID-19 drug interactions resource. Of interest, only 10 DDI pairs were recognized by both of these drug interaction resources. Conclusion: Clinicians should not entirely rely on any individual DDI resource for checking life threatening ADRs of LPV/RTV in patients with COVID-19 since there were discrepancies in enlisting contraindicated drugs.
\end{abstract}

\section{What is already known about this subject}

- Lopinavir (LPV)/Ritonavir (RTV) may potentially interact with contraindicated medications affecting its metabolism and may produce life-threatening adverse drug reactions (ADRs) due to drug-drug interactions (DDIs).

- Potential clinically significant life-threatening DDIs of this combination antiviral therapy used in the treatment of COVID-19 patients has not systematically reviewed yet.

\section{What this study adds}

- In total, 62 severe DDI pairs of LPV/RTV as identified from the FDA and Liverpool DDI resources should be given serious clinical considerations predicted to cause life threatening ADRs.

- Of interest, only 10 severe DDI pairs were recognized by both the FDA and Liverpool DDI resources, further emphasized to undertake systematic cross-comparisons between DDI resources in order to identify discrepancies and possibly avoid any interacting drugs that may cause life-threatening ADRs of LPV/RTV in COVID-19 patients.

- Clinicians should not entirely rely on any individual DDI resource for checking life threatening ADRs of LPV/RTV in patients with COVID-19 since there were disagreements in enlisting contraindicated drugs.

\section{Introduction}

It is identified in the literature that considerable proportion of patients infected with severe acute respiratory syndrome corona virus-2 (SARS-CoV-2) are using combination of lopinavir (LPV) and ritonavir (RTV) as first line antiviral therapy for the treatment of this pandemic virus ${ }^{1}$. It is also notable that many patients 
with COVID-19 had multiple comorbidities and are vulnerable to polypharmacy ${ }^{2,3}$ which may in turn leads to DDIs due to taking multiple medications. A recent clinical trial of LPV/RTV didn't find superiority of using this combination in COVID-19 patients ${ }^{4}$. Although many factors e.g. age, sex, comorbidities, clinical features etc. might trigger the clinical outcomes, however, one of the major contributing factors of these may be partly due to the DDIs associated with COVID-19 patients due to having multiple comorbidities.

Being potent inhibitors of CYP3A4 enzyme, LPV/RTV can exert uniquely powerful inhibiting effects of CYP3A4 substrate drugs, potentially increased blood concentrations of substrate drugs which may leads to a number of clinically significant DDIs ${ }^{5,6}$. Contrastingly, CYP3A4 inducer drugs may expedite the hepatic clearance of LPV/RTV and may cause therapeutic failure leading to reduced virologic control ${ }^{7}$. Being acting as substrates of a transporter protein called permeability glycoprotein $(\mathrm{P}-\mathrm{gp})^{8}$ known as efflux transporter, the pharmacokinetics (PK) effects of LPV/RTV may also be affected by the co-prescription with substrate, inhibitor or inducer drugs of P-gp and were predicted to cause potential clinically significant DDIs.

However, the FDA prescribing information of LPV/RTV indicated that this combination therapy is 'contraindicated with drugs that are highly dependent on CYP3A4 for clearance and for which elevated plasma concentrations are associated with serious and/or life-threatening reactions'. Also, it is 'contraindicated with drugs that are potent CYP3A4 inducers where significantly reduced lopinavir plasma concentrations may be associated with the potential for loss of virologic response and possible resistance and cross-resistance ${ }^{9}$.

On the other hand, the Liverpool interactions group has provided prescribing resources where they categorized the interactions of experimental COVID-19 antiviral therapies as 'contraindicated medications', 'potential interactions requiring dose adjustment/close monitoring', 'potential interactions of weak intensity' or 'no clinically significant interactions ${ }^{10}$. Contraindicated medications should not be coadministered concurrently due to potential life-threatening ADRs.

Amid this emergency situations, details of the treatments provided in COVID-19 patients and consequent clinical outcomes were not available and was therefore unable to assess either the actual ADRs/drug toxicity or potential clinically significant DDIs. It is also pertinent to noted that while in many countries especially in developed countries, DDI alert systems are functional, however, one of the major problems of implementing DDIs assessment in clinical practice is that most clinicians become fatigue to DDI alerts and majority of the time the alerts are overridden due to too much warnings ${ }^{11}$.

Therefore, the present study was aimed to predictively identify severe DDIs pairs only predicted to cause life-threatening ADRs from international resources so as to aware clinicians regarding the severity of these interactions.

\section{Methods}

To identify potential clinically significant life-threatening DDIs, this study used the U.S. Food and Drug Administration (FDA) prescribing table of LPV/RTV enlisted contraindicated medications ${ }^{9}$. Likely, potential clinically significant life-threatening DDIs were also identified from the lists of contraindicated drugs of LPV/RTV as provided by the Liverpool COVID-19 drug interaction resource ${ }^{10}$. The interacting contraindicated drugs were putatively paired with LPV/RTV called severe DDI pairs predicted to cause life-threatening ADRs.

The severe DDI pairs identified from the FDA and Liverpool COVID-19 interaction resources were then analyzed using a Venn diagram showing the unique or overlapping drug pairs between these two well recognized evidence-based international DDI resources.

\section{Results}

After removing duplicates, in total of 62 severe DDI pairs of LPV/RTV were identified from the FDA and Liverpool COVID-19 interaction resources predicted to cause clinically significant life-threatening ADRs, as shown in Figure 1. Of these, seven unique DDI pairs were identified from the FDA only whereas 45 unique DDI pairs were identified from the Liverpool COVID-19 drug interactions resource. Interestingly, only 10 
DDI pairs were recognized by both of these drug interaction resources. The lists of all interacting drugs as identified from these two DDI resources are shown in Table 1.

Systematic cross-comparison between these two well-recognized evidenced-based DDI resources indicated that discrepancies were existed in between these two resources for enlisting contraindicated drugs potentially causing life-threatening ADRs of LPV/RTV.

Severe DDI pairs that were not enlisted by the Liverpool COVID-19 DDI resource and the potential lifethreatening ADRs are tabulated in Table 2 as identified from the FDA prescribing information of LPV/RTV. Notable life-threatening ADRs due DDIs of LPV/RTV with these drugs were cardiac arrhythmias, vasospasm, ischemia and hypotension.

Severe DDI pairs that were recognized by both the FDA and the Liverpool COVID-19 DDI resource and the potential life-threatening ADRs are tabulated in Table 3. Notable life-threatening ADRs due DDIs of LPV/RTV with these drugs were cardiac arrhythmias, myopathy, rhabdomyolysis, respiratory depression, risk of alanine transaminase (ALT) elevations, hypotension and risk of reduced virologic response.

In summary, Liverpool COVID-19 DDI resource was more effective in enlisting contraindicated drugs of LPV/RTV potentially causing life-threatening ADRs, however, clinicians should not entirely rely on this resource for checking DDIs of LPV/RTV since it didn’t enlist several important contraindicated drugs of LPV/RTV predicted to cause life-threatening ADRs.

\section{Discussion}

This is the first study assessing severe DDI pairs of LPV/RTV used in the treatment of COVID-19 patients and were predicted to cause clinically significant life-threatening ADRs.

The findings of this study have great novelty in terms of reducing clinician burdens especially when DDI alert fatigues may expected to occur in this emergency pandemic health situations. This study has tabulated the composite lists of severe DDI pairs of LPV/RTV that were recognized by both the international wellrecognized evidenced-based resources and is highly recommended to check at least 10 severe DDI pairs of LPV/RTV to reduce the risk of life-threatening ADRs.

Meanwhile, it is also noteworthy that in many developing countries, computer based DDI alert systems may not available and clinicians in these countries may be advantaged greatly from the severe DDI pairs identified in this study in general, and in particular the composite list recognized by both the international drug interactions resources.

Important findings of this study revealed that clinicians should not depend on any particular DDI checking systems of LPV/RTV and may be other COVID-19 drugs as well since high level of discrepancies were identified in this study. For example, if clinicians solely rely on the Liverpool COVID-19 DDI checking system which has recently emerged specifically for COVID-19 therapeutics, it is found that around seven very clinically important drugs may be missed for checking DDIs that may cause life-threatening ADRs. On the other hand, if clinicians solely rely on the FDA prescribing information of LPV/RTV, it is found that around 45 very clinically important drugs may be missed for checking DDIs that may also cause lifethreatening ADRs. Therefore, it is suggested to undertake systematic cross-comparison in future by the professional DDI bodies to reduce disagreement in between the drug interaction resources. Also, suggested to identify any discrepancies by the clinicians in order to avoid missing any interacting drugs predicted to cause clinically significant DDIs.

It is important to recognize that although different other ways e.g. computerized DDI alerts ${ }^{12,13}$, web-based DDI checkers etc. can be used to identify potential clinically significant DDIs of LPV/RTV, however there are lots of limitations of these checking systems including alert fatigues ${ }^{11}$, lack of robust evidence of the interactions etc. The findings of the present study may therefore serve as considerably best severe DDI pairs of LPV/RTV predicted to cause life-threatening ADRs since the DDI pairs were identified from the internationally well-recognized evidenced based DDI resources. 


\section{Future implications of potential severe DDI pairs of LPV/RTV}

Amid this emergency situations, it is highly suggested to check severe DDI pairs of LPV/RTV as identified in this study and were predicted to cause clinically significant life-threatening ADRs in patients with COVID-19 for optimizing its safety or efficacy. It is also effective to implement these severe DDI pairs of LPV/RTV to identify potential clinically significant DDIs in other disease conditions where it is clinically indicated.

\section{Conclusions}

The findings of this study suggest to consider 62 putative severe DDI pairs of LPV/RTV identified from two international evidenced-based drug interactions resources predicted to cause clinically significant lifethreatening ADRs in COVID-19 patients. It is also suggested that systematic cross-comparisons may help to identify discrepancies in between the drug interaction resources. Further, it is highly suggested that clinicians should not entirely rely on any individual DDI resource for checking life threatening ADRs of LPV/RTV in patients with COVID-19 since there were disagreements in enlisting contraindicated drugs.

Conflict of interest: No conflict of interest.

Funding: No funding was available.

Data availability: All data used in this analysis are available upon request.

\section{References}

1. Yousefifard M, Zali A, Mohamed Ali K, et al. Antiviral therapy in management of COVID-19: a systematic review on current evidence.Arch Acad Emerg Med . 2020;8(1):e45. http://www.ncbi.nlm.nih.gov/pubmed/32309809.

2. Richardson S, Hirsch JS, Narasimhan M, et al. Presenting Characteristics, Comorbidities, and Outcomes Among 5700 Patients Hospitalized With COVID-19 in the New York City Area. Jama . 2020. doi:10.1001/jama.2020.6775

3. Bessière F, Roccia H, Delinière A, et al. Assessment of QT Intervals in a Case Series of Patients with Coronavirus Disease 2019 (COVID-19) Infection Treated with Hydroxychloroquine Alone or in Combination with Azithromycin in an Intensive Care Unit. JAMA Cardiol . 2020. doi:10.1001/jamacardio.2020.1787

4. Cao B, Wang Y, Wen D, et al. A Trial of Lopinavir-Ritonavir in Adults Hospitalized with Severe Covid-19. N Engl J Med . 2020. doi:10.1056/nejmoa2001282

5. Francis J, Barnes KI, Workman L, et al. An individual participant data population pharmacokinetic meta-analysis of drug-drug interactions between lumefantrine and commonly used antiretroviral treatment.Antimicrob Agents Chemother . 2020;64(5). doi:10.1128/AAC.02394-19

6. Food and Drug Administration. Drug Development and Drug Interactions: Table of Substrates, Inhibitors and InducersNo Title. https://www.fda.gov/drugs/drug-interactions-labeling/drug-development-and-druginteractions-table-substrates-inhibitors-and-inducers\#table3-1. Published 2020. Accessed June 15, 2020.

7. Hughes CA, Tseng A, Cooper R. Managing drug interactions in HIV-infected adults with comorbid illness. Cmaj . 2015;187(1):36-43. doi:10.1503/cmaj.131626

8. Liu X, Ma Q, Yan Z, et al. Impact of Single Nucleotide Polymorphisms on Plasma Concentrations of Efavirenz and Lopinavir/ritonavir in Chinese Children Infected with the Human Immunodeficiency Virus.Pharmacotherapy . 2017;37(9):1073-1080. doi:10.1002/phar.1988

9. FDA. Highlights of prescribing information of lopinavir/ritonavir. https://www.accessdata.fda.gov/drugsatfda_docs/label/2013/021226s038lbl.pdf. Published 2020. Accessed June 2, 2020. 
10. University of Liverpool. Detailed recommendations for interactions with experimental COVID-19 antiviral therapies. https://www.covid19-druginteractions.org/prescribing-resources. Published 2020. Accessed June $4,2020$.

11. Nanji KC, Seger DL, Slight SP, et al. Medication-related clinical decision support alert overrides in inpatients. J Am Med Inform Assoc . 2018;25(5):476-481. doi:10.1093/jamia/ocx115

12. Zenziper Straichman Y, Kurnik D, Matok I, et al. Prescriber response to computerized drug alerts for electronic prescriptions among hospitalized patients. Int J Med Inform . 2017;107:70-75. doi:10.1016/j.ijmedinf.2017.08.008

13. McEvoy DS, Sittig DF, Hickman TT, et al. Variation in high-priority drug-drug interaction alerts across institutions and electronic health records. J Am Med Informatics Assoc . 2017;24(2):331-338. doi:10.1093/jamia/ocw114

\section{Hosted file}

Figure.docx available at https://authorea.com/users/338169/articles/464174-potentialclinically-significant-life-threatening-drug-drug-interactions-of-lopinavir-andritonavir-used-in-the-treatment-of-covid-19

\section{Hosted file}

Tables.docx available at https://authorea.com/users/338169/articles/464174-potentialclinically-significant-life-threatening-drug-drug-interactions-of-lopinavir-and-

ritonavir-used-in-the-treatment-of-covid-19 